\title{
Características familiares de adolescentes consultantes y no consultantes en unidades de salud mental
}

\author{
Arturo Roizblatt S. '; Macarena Valdés C. '; Ramón Florenzano U. '; Jorge Rodríguez A.' ; \\ Teresita Seriano G.' ; Juan F. Labra J. '; Pablo Lara P..'; Marcela López P.'
}

\begin{abstract}
Resumen
Propósino: determirar si exișten diterencicias en coracterísticas de función famililior que permilen predecir conduelas de riesgo para la solud menta: en odolescentes, asi como ciras que constituyer criterios de estas jllimas. Ambíto: adolestentes que consultan en unidades públicas de aención de sa:ud merital y coe-áneos no consu tantes alumnos de establecimientos educacionales, anbos de Sant cgo mejropo itano Parsonas y métodc: 508 familias con -ijas adoles. centes ro consultantes y 127 tamilias con ricos adalescentes consultartes en unidacies de salud mental, fueron encuestadas con las correspondiertes versiones sora adolescente y postres de un instrumerto que incluye seis "ac'ores de predicción y cinco de er lerio para conciuctas de riesgo de la salud de los adolescentes. Resuttados: En el grupo de las adolescentes consultanles se encontró significotivamente maycr conjidad de factores de riesgo paro la salud lsegún padres: ventaja $\mathrm{C}, 47 ; p<0.001$ i y pat:ones meros polectores. con percepciones deficiertes especialmente en cominicaciones familiares [segin acolescentes: ventoja 0.93; $P<0,002$ ], estilos de soluciún de protlenas [segin podres ventajo 1,16: $p<0,002$ : según aco escentes: ventá $0.88: p<0,03$ i y d sponibi idad de recursos familiares externos |según oddescentes: ventaja 0.87: $p<0.02$ ) en el inter or de la fami io.
\end{abstract}

[Palabras clave: acolescenc a, función forriliar, solud merilal.]

\section{Family functional aspects among adolescents asking or not asking for mental health care}

Otiecrive 10 delermine if there cre differences in functional fomily factors prediclive 0 constitutive of risks for healtr umong adolescents osking for menlol heo th core. Design: case contro! Selting: urban generol communily, government realth acilties. melropolitan Sentiage, Chile. Porticipants: codolescents seeking care for mento health problems in: $\vdots 27$ ! cge malched public schools students never asking for menial tealth care Im; 5081, and heir correspencing torrilies. Outcome measures. participants we'e asked to respeclively fill either ar acolescent or a parental version of $c$ questionncire on 'ami.y functicn aspecls considered 0 be predictve [six) or constitutive (fivel of healis risk. Resurits. acolescent hed th risk factors ifarens OR 0.47. $\mathrm{p}<0.001$ i and less protective functiona fartly patterns were sigrificontly more fequently eported by subjects seekng cars and their parents, particularily in tonly communica:ion |adolescenls OR $0.93, p<0.002$, and problerr selving sty es iparen's $O R$ I l $6, p<0.002$, acolescents $O R 0.89$, $p<0.03$ ), and avoilability of exeno famly resources (addescenls OR. 0.87, $p<0.02$ ).

(Key words: adolescent, menta heclin problerrs, family funtion.)

La salud del adolescente es motivo de creciente preocupación. La influencia de la estructura y funcionalidad de la familia sobre el bienestar de sus integrantes ha sido sujeto de estudios del

1. Departantento de Psiquiatría, Unidad de Salud Mental. Facultad de Medicina, Campus Oriente, Universidad de Chile.

Trabajo prisparado dentro del proyecto FONDECYT $N^{2} 1940277$. área de la salud y de las ciencias sociales'. La familia puede constituir tanto un ente protector como una fuente de factores de riesgo (cuando existen ciertas características de disfuncionalidad), para el adolescente $2-5$. Lo anterior justifica la preocupación por estudiar y medir la funcionalidad familiar y su capacidad de otorgar protección a sus miembros contra conductas de riesgo para su bienestar ${ }^{6-B}$.

Con el objeto de medir la funcionalidad familiar en sus diferentes aspectos se han disefiado 
instrumentos para ser aplicados a los adolescentes y a los padres. Ellos han sido ampliamente analizados en el marco de un proyecto internacional de la $\mathrm{OMS}^{9}$. Con propósitos de comparación, se aplicaron estos instrumentos a dos grupos de adolescentes y a sus respectivos padres: uno formado por adolescentes que consultaron en unidades ambulatorias (consultorios) u hospitalarias de salud mental y otro de adolescentes no consultantes (alumnos de diversos colegios de Santiago), en busca de conductas protectoras y de riesgo y características de funcionalidad familiar que pudieran explicar dichas conductas.

\section{Material y Método}

Se tomó una muesira aleatoria estratificada, representativa de la población de familias con hijos adolescentes de Santiago, consistente en 635 familias beneficiarias del sector publico o municipal del área metropolitana: 508 de adolescentes no consultantes y 127 de adoJescentes consultantes en unidades de salud mental, que to hicieton principalmente por trastomos patemo-filiales, de adaptación. de identidad, ansiedad, $\mathrm{u}$ ottos ${ }^{/ 11}$. Los criterios de estratificación para la muestra fueron: comuna (según clasificación socioeconómica), establecimiento educacional y curso para los no consultartes y consultorio u hospital para los consultantes

Los adolescentes no consultantes, una vez seleccionados los colegios y cursos, fueron encuestados en caùa estahlecimiento educacional. En el caso de los adolescentes tonsultantes, se encuesto a quienes asistieron en los meses de junio. julio, septienbre y octubre de 1995 en los consultorios u hospitales seleccionados. Los padres de los primeros fueron entrevistados en las reuniones de apoderados en los colegios o -en caso de inasistencia-fueron visitados en sus hogares, Los padres de los consultantes fueron citados al consultorio u hospital, donde se les pidió responder el cuestionario.

En ambos grupas se aplicaron dos fornatos, respectivamente. destinados uno a consultar a los adolescentes ( $¿$ Como es tu familia?") y otro a los padres ("¿Como es su familia?"). del instrumenco de medición". En caso de los padres la respuesta se pidió alealoriamente al padre o la madre.

Para comparar las mediciones entre consultantes y no consultantes se utilizaron cinco factores de criterio (conjunto de variables que agtupadas forman una conducta que se desea predecir) y seis de predicción (conjunto de variablés que agrupadas intentan explicar o anticipar una conductai en ambas versiones del cuestionario (para padres y para adolescentes), los que fueron seleccionados luego de un análisis factorial ${ }^{4}$. aplicando el método estadísico de regresión Iogística

\section{Resultados}

Se encuestaron 635 familias, 508 de adolescentes no consultantes (edad promedio 14,1 años, $32 \%$ mujeres) y 127 de consultantes (edad promedio 15,1 años, $55 \%$ mujeres) en unidades ambulatorias u hospitalarias de salud mental. En $95 \%$ de los casos de ambos grupos de jóvenes la madre bjológica vivía en el hogar, mientras el padre biológico lo hacía en $83,3 \%$ de las familias de no consultantes y $66,1 \%$ de los consultantes (tabla 1).

Casi todas las familias encuestadas contaban con servicios básicos de agua potable y luz eléctrica. Tenían vivienda propia $39,7 \%$ de las de no consultantes y $53,2 \%$ de las de consultantes. En $42,6 \%$ de los adolescentes no consultantes el ingreso familiar mensual declarado era inferior a dos salarios mínimos y en $22 \%$ mayor a cinco salarios mínimos, siendo las correspondientes proporciones entre jốvenes consultantes de $66,1 \%$ y $7,4 \%$ respectivamente.

Se encontraron diferencias estadísticamente significativas entre las respuestas de los padres de adolescentes consultantes y no consultantes en los siguientes tres de los seis factores predictores incluidos: "Comunicación conyugal", "Disponibilidad de recursos" y "Estilo familiar de resolución de problemas". Las respuestas de los adolescentes mostraron diferencias signifi-

\section{Tabla 1}

Características generales de familias de adolescentes consultantes y no consultantes en unidades de salud mental

(Santiago 1995)

\begin{tabular}{lrr}
\hline Características & No cons. & Cons. \\
\hline Edad (unios, $\bar{x}$ ) & 14,1 & 15,1 \\
Integrantes familia $(\overline{\mathrm{x}}$ ) & 3,9 & 3,9 \\
Hijos $(\overline{\mathrm{x}})$ & 2,3 & 2,9 \\
Hombes (\%) & 67,9 & 44,9 \\
Mujeres (\%) & 32,1 & 55,1 \\
Hijo único (\%) & 9,1 & 14,3 \\
Madre en el hogar (\%) & 95,9 & 95,2 \\
Padre en el hogar (\%) & 83,3 & 66,1 \\
Pareja del padre (\%) & 8,7 & 5,8 \\
Pareja de la madre (\%) & 12,7 & 20.7 \\
\hline
\end{tabular}

Cons: consultante. 
cativas en los factores "Comunicación y rituales familiares". "Estilo familiar de resolución de problemas" y "Disponibilidad de recursos externos" (tabla 2). Esta incompleta equivalencia entre las respuestas de los padres y los adolescentes queda ilustrada en el hecho de percibir, los jóvenes consultantes, mayores problemas en la comunicación familiar global, mientras sus padres lo hacen en mayor medida en su comunicación conyugal. Del mismo modo, mientras los jovenes perciben dificultades en la disponibilidad de recursos próximos a la farnilia para enfrentar la vida y sus problemas, tales como hermanos, parientes cercanos y amigos, sus padres entocan los problemas en la disponihilidad de recursos externos a la familia, como programas comunitarios, profesionales de distintas áreas, sacerdotes. En cambio se registró concordancia entre padres y adolescentes en la percepción de alteraciones en el estilo familiar de resolución de problemas, y diferencias significativas entre consultantes y no consultantes en las respuestas de los dos componentes familiares: por ejemplo, los adolescentes consultantes y sus padres aludieron a dificultades para expresar lo que sc desea al interior de la familia $(36,3 \%)$, falta de consideración a las ideas de los hijos $(50,3 \%)$, falta de unión para enfrentar las dificultades $(49,6 \%)$ y ausencia de definiciones optimistas de los problemas $(43,3 \%)$.
Sc registraron diferencias estadísticamente significativas en dos de los cinco factores de criterio entre las respuestas de los padres: "Riesgo y salud del adolescente" y "Salud mental del adolescente", mientras en las de los adolescentes se encontró diferencia con significación estadística en uno de los cinco factores: "Salud mental adolescente y familiar" (tabla 3). Al integrar las respuestas de los padres y adolescentes, la diferencia más importante entre adolescentes consultantes y no consultantes fue la presencia de distintos factores y conductas de riesgo y sus posibles consecuencias en alteraciones de su salud física y mental, que los llevan a consultar y buscar aigún tipo de tratamiento (tabla 4). En la misma tabla se observa que el antecedente de haber sufrido maltrato físico y los sentimientos de tristeza y ansiedad son dos veces más frecuentes en adolescentes consultantes, según ellos lo indican, y el abuso sexual casi cuatro veces más.

Algunas conductas de riesgo como fumar, beber alcohol hasta embriagarse o consumo de marihuana, muestran una mínima mayor frecuencia en adolescentes consultantes, mientras el uso de otras drogas se registró en las respuestas de $3,2 \%$ de los adolescentes y $1,2 \%$ de los padres del grupo no consultantes, lo que aumenta Jrásticamente a $23,6 \%$ en las de los jóvenes consultantes y $21,8 \%$ en las de sus padres.

\section{Tabla 2}

Factores predictivos de conductas de riesgo en las versiones para adultos y para adolescentes de la encuesta de funcionamiento familiar

\begin{tabular}{|c|c|c|c|c|}
\hline \multirow{2}{*}{ Factor } & \multicolumn{2}{|c|}{ Adultos } & \multicolumn{2}{|c|}{ Adolescentes } \\
\hline & Ventaja & $\mathrm{p}$ & Ventaja & $\mathbf{p}$ \\
\hline Conunicación conyugal & $0,86^{*}$ & $<0,002$ & - & - \\
\hline Comunicacion roles y rituales & 0,95 & $>0.270$ & $0,93^{*}$ & $<0,002$ \\
\hline Valores y creencias & 0,94 & $>0.140$ & 0,95 & $>0.330$ \\
\hline Resolucion de problemas & $1,16^{x}$ & $<0,002$ & $0,88^{*}$ & $<0,030$ \\
\hline Recursos próximos & $0.88^{-}$ & $<0,020$ & 1,08 & $>0,390$ \\
\hline Recursos familiares externos & 0,99 & $>0.810$ & $1,16^{*}$ & $<0,020$ \\
\hline Jerarquia y poder & - & - & 0,87 & $>0.220$ \\
\hline
\end{tabular}

Ventaja: razón de productos cruzados u "odds ratio" (inglés).

* Estadísticamente significativo. 


\section{Tabla 3}

Factores de criterio de riesgo en las respuestas a las versiones para adolescentes y adultos de la encuesta de funcionamiento familiar, comparación entre consultantes y no consultantes

\begin{tabular}{|c|c|c|c|c|}
\hline \multirow{2}{*}{ Factor } & \multicolumn{2}{|c|}{ Adultos } & \multicolumn{2}{|c|}{ Adolescentes } \\
\hline & Ventaja & $\mathbf{p}$ & Ventaja & P \\
\hline Riesgo y salud familiar & 0,94 & $>0,670$ & 0,98 & $>0,850$ \\
\hline Riesgo y salud adolescente & $0,47 *$ & $<0,001$ & 0,65 & $>0,100$ \\
\hline Satisfacción calidad de vida & 0,92 & $>0.190$ & 0,95 & $>0,160$ \\
\hline Salud mental adolescente & $0.24^{*}$ & $<0,001$ & - & - \\
\hline Autoimagen familiar & 1,06 & $>0,800$ & - & - \\
\hline Salud mental adolescente $y$ familia & - & - & $0,61 *$ & $<0,00]$ \\
\hline Conductas impulsivas & - & - & 1,14 & $>0,360$ \\
\hline
\end{tabular}

Ventaja: razón de productos cruzados, "odds ratio"

* Estadisticamente significativo.

Tabla 4

Descripción de algunas variables incluidas en los factores criterio de mayor relevancia de acuerdo con las respuestas de padres y adolescentes de grupos de consultantes y no consultantes

\begin{tabular}{|c|c|c|c|c|}
\hline \multirow{2}{*}{ Variables } & \multicolumn{2}{|c|}{ Adultos } & \multicolumn{2}{|c|}{ Adolescentes } \\
\hline & $\begin{array}{c}\text { No cons. } \\
(\%)\end{array}$ & $\begin{array}{l}\text { Cons. } \\
(\%)\end{array}$ & $\begin{array}{c}\text { No cons. } \\
\text { (\%) }\end{array}$ & $\begin{array}{l}\text { Cons. } \\
(\%)\end{array}$ \\
\hline Hijo triste o nervioso & 26,8 & 64,2 & 29.4 & 59.8 \\
\hline Otro triste o nervioso & 32,8 & 63,1 & 27,9 & 51.2 \\
\hline Hijo sufrío abuso sexual & 0,0 & 4,1 & 1.4 & 5.5 \\
\hline Hijo sufrió golpes o maltrato & 6,4 & 22,8 & 7,5 & 14.2 \\
\hline Hijo drogas, no marihuana & 1,2 & 21.8 & 3,2 & 23,6 \\
\hline Hijo intento suicidjo & 2,2 & $2 J, 1$ & 8,3 & 21,3 \\
\hline Hijo cons. por probl. psicologicos & 14,4 & 69,4 & 15,1 & $6 \mathrm{~L}, 4$ \\
\hline Hijo Iratam. psicol o psiquiatr. & 5,3 & 54,4 & 6,8 & 56,7 \\
\hline Otro cons. por probl. psicologicos & 23,6 & 58.2 & 22.8 & 40,5 \\
\hline
\end{tabular}

Cons: consultante. 
Otras consecuencias de conductas riesgosas como la actividad sexual precoz, son el embarazo y el aborto en adolescentes. Las respuestas de los padres informaron que el embarazo es aproximadamente cuatro veces mas frecuente entre adolescentes consultantes que en no consultantes $(11,3 \%$ ante $2,4 \%)$ y el aborto dos veces más frecuente $(1,6 \%$ ante $0,8 \%)$. Del mismo modo destaca la mayor proporción de intentos de suicidio que refieren los propios adolescentes. Esta cifra tan alta en parte podría explicarse argumentando que los adolescentes pueden incluir como intento, conductas no estrictamente suicidas o tan sólo la ideación suicida. Sin embargo la concordancia con lo expresado por sus padres podría indicar que el dato es real, en el sentido comentado.

Por tratarse éste de un análisis sobre adolescentes consultantes en unidades de salud mental, es logico encontrar altos porcentajes de consulta por problemas psicológicos o psiquiátricos y de haber recibido algún tipo de tratamiento, sin embargo, es de hacer notar que $15 \%$ de los jóvenes no consultantes y $22,8 \%$ de los otros miembros de la familia declaren haber consultado por algún problema de esta índole.

En la tabla 5 se destacan las características diferenciales más ostensibles entre adolescentes consultantes y no consultantes, sobre todo las aparentes dificultades en la comunicación entre padres e hijos y en la relación con parientes cercanos.

\section{Comentario}

En lo que respecta a los factores definidos como criterio, que contienen eleruentos que buscan detectar factores y conductas de riesgo, así como consultas y tratamientos recibidos por el adolescente y su familia como consecuencia de los anteriores, las diferencias obtenidas entre no consultantes y consultantes señalan que los primeros presentan frecuencias de factores y conductas de riesgo, como también de tratamientos recibidos, claramente mayores que los no consultantes, lo que justifica el estudio de sus modelos de funcionalidad familiar, si se está de acuerdo en que ellos constituyen uno de los factores determinantes.

Las diferencias en las respuestas de padres e hijos, que podrian explicarse en parte por mini-

\section{Tabla 5}

Descripción de algunas variables incluidas en los factores de predicción de mayor relevancia, según las respuestas de adolescentes consultantes y no consultantes

\begin{tabular}{lcc}
\hline & \multicolumn{2}{c}{ Adolescentes } \\
\cline { 2 - 3 } Variables & $\begin{array}{c}\text { No cons. } \\
\%\end{array}$ & $\begin{array}{c}\text { Cons. } \\
\%\end{array}$ \\
\hline $\begin{array}{l}\text { No satisfecho como conversa } \\
\text { con la madre }\end{array}$ & 9,0 & 20,6 \\
$\begin{array}{l}\text { No satisfecho como conversa } \\
\text { Con el padre }\end{array}$ & 19,5 & 34,9 \\
$\begin{array}{l}\text { El padre no habla a diario } \\
\text { con los bijos }\end{array}$ & 49,0 & 65,0 \\
$\begin{array}{l}\text { No satisfecho con la relacion } \\
\text { con parientes }\end{array}$ & 13,4 & 30,0 \\
$\begin{array}{l}\text { No les gusta pasar juntos } \\
\text { el tiempo libje }\end{array}$ & 6,2 & 18,9 \\
$\begin{array}{l}\text { No se expresa fácilmente } \\
\text { lo que se desea }\end{array}$ & 18,5 & 36,3 \\
\hline
\end{tabular}

Cons: consultante.

mización o negación por parte del adolescente sobre ciertos problemas que lo afectan o sobreestimación de los mismos por los padres, muestran, sin embargo, diferentes percepciones de las situaciones intrafamiliares entre ambos componentes, por lo que encuestar a padres e hijos adolescentes por separado, con dos versiones del mismo instrumento, es fundamental para lograr un enfoque global y complementario. Los factores predictores señalan pues que en los adolescentes consultantes se encuentran más características de disfuncionalidad familiar, consideradas no protectoras, lo cual podría explicar, en mayor o menor medida, conductas de riesgo para su salud.

A la luz de estos resultados, se considera necesario desarrollar programas preventivos que promuevan la adecuada comunicación en el interior de la familia, fomenten la adquisición de destrezas para enfrentar y resolver los problemas familiares, ayudando a los adolescentes y sus familias a reconocer y revertir los trastornos funcionales de éstas. 


\section{Referencias}

1. Valdes M, Serrano T. Florenzano R. et al: Factores familiares protecrores para conductas de riesgo: vulnerabilidad y resiliencia adolescentes. Rev Psquiatra Clín 1995: 32: 49-56.

2. Ruter $M$ : La resiliencia: Consideraciones conceptuales. Elsevier Science Pubtishing Co. Inc. New York. 1993.

3. Dreyfors J: Adolescents at risk, prevalence and prevention. New York, Oxford Universily Press, 1990.

4. MiEkduhl $M$. Rirc $R$, Shuts $\boldsymbol{W}$ : Children of parents hospitalized for tnental illness. Am J Pub Health 1962; 52: 428-435.

5 Florenzano R. Marchandon A. Pino P: Frecuencia de consumo de sustancias químicas y de conductas antisociales en adolescentes hijos de padres con psicopato- logía mayor. XLVII Jotnadas Soc. Neurologia, Psiquiatria y Neurocirugía 1992.

6. Olson D. Portner J. Lave Y: Escalas de evaluación de adaptabilidad y cohesión familiar (FACES Jll). Handbook of measurements for marriage and family therapy. New York, Brunner Mazel, 1987.

7. Maddaleno M. Horwitz $N$, Jara $C$, Flarenzanto $R$, Salazar D: Aplicación de un insurumento para calificar e) funcionamiento familiac en la atención de adolescentes. Rev Chil Pediatr 1987; 58: 246-249.

8. Alvarez $M$ : La familia en riesgo: un instrumento de dingnóstico. Edic. Unjversitaria Santiago de Chile 1986.

9. Rodríguez J. Serrano $T$, Vaides $M$, er al: Andilisis de la confiabilidad $y$ validez de un instrumento que mide el rol protector familiar en las conductas de riesgo adolescente. Cuademos Médico Sociales 1996; 37: 64-89.

Esta publicación está disponible en copias de microfilms de $16 \mathrm{y}$ $35 \mathrm{~mm}$ y microfichas de $105 \mathrm{~mm}$, las que pueden solicitarse a: University Microfilms International

300 North Zeeb Road

Ann Arbor, Michigan 48106, USA.

This journalis also available in $16 \mathrm{~mm}$ microfilm, $35 \mathrm{~mm}$ microfilm and $105 \mathrm{~mm}$ microfilm copies through

University Microfilms International,

300 North Zeeb Road,

Ann Arbor, Michigan 48106, USA. 